ANNALES

POLONICI MATHEMATICI

$91.1(2007)$

\title{
Universal divisors for Hardy spaces in the polydisk
}

\author{
by Michat JasiczaK (Poznań)
}

\begin{abstract}
We show that for an interpolating sequence in the polydisk one can construct a universal divisor for Hardy spaces.
\end{abstract}

1. Introduction. It is a remarkable fact in one variable Hardy space theory that given a function $f \in H^{p}(\mathbb{D})$, one can find another function $g \in H^{p}$ with no zeros in $\mathbb{D}$ such that $f=B g$. Here $B$ stands for the Blaschke product for the zero set of $f$. What is important here is that $B$ depends only on the zeros of $f$, not on $f$ itself. That is why the Blaschke products are called universal divisors for the Hardy space. Let us emphasize that the Blaschke product is a product of automorphisms of the unit disk.

The situation in several variables is more involved. No such result is known for a general domain $D \subset \mathbb{C}^{n}$. However, in some cases one can try to obtain similar results. Importantly, for this idea to work one needs the group of automorphisms of $D$ to act transitively on $D$. In $\mathbb{C}^{n}, n>1$, there is only one domain with this property and $C^{2}$ boundary - the unit ball $\mathbb{B}$ (cf. [9]). The question of existence of universal divisors in Hardy spaces in the case of the unit ball was investigated in [3]. It was shown that one can construct such divisors for a discrete set $\mathcal{Z} \subset \mathbb{B}$, under the assumption that $\mathcal{Z}$ is an interpolating sequence for Hardy spaces.

In this paper we study another standard domain in $\mathbb{C}^{n}$, the polydisk. We prove the following fact:

THEOREM 1. If $\mathcal{Z}$ is an interpolating sequence for $H^{\infty}\left(\mathbb{D}^{n}\right)$ in the polydisk, then there is a universal divisor (of dimension $n+2$ ) for $\mathcal{Z}$ and any $H^{p}$ with $2<p<\infty$.

The next definition specifies what we mean by a universal divisor.

Definition 1. We shall say that $B=\left(B_{1}, \ldots, B_{N}\right) \in\left(H^{\infty}\right)^{N}$ is a universal divisor (of dimension $N$ ) for $\mathcal{Z}$ and the space $H^{p}$ if $\left.B\right|_{\mathcal{Z}}=0$ and

2000 Mathematics Subject Classification: Primary 32A35; Secondary 32A10, 32A60.

Key words and phrases: Hardy space, polydisk, interpolating sequence, universal divisor, Carleson measure. 
for any function $f \in H^{p}$ with $\left.f\right|_{\mathcal{Z}}=0$, there is $F \in\left(H^{p}\right)^{N}$ such that

$$
f=\sum_{j=1}^{N} F_{j} B_{j} .
$$

The polydisk has a transitive group of automorphisms. Let $\Phi_{z}, z=$ $\left(z_{1}, \ldots, z_{n}\right) \in \mathbb{D}^{n}$, denote the biholomorphism

$$
\Phi_{z}(\zeta)=\left(\frac{z_{1}-\zeta_{1}}{1-\bar{z}_{1} \zeta_{1}}, \ldots, \frac{z_{n}-\zeta_{n}}{1-\bar{z}_{n} \zeta_{n}}\right) .
$$

We shall use the terminology from [3].

DeFinition 2. Let $\mathcal{Z}$ be a sequence of points in $\mathbb{D}^{n}$. We shall say that a $\mathbb{C}^{N}$-valued bounded holomorphic function $B=\left(B_{1}, \ldots, B_{N}\right)$ is an $N$ strongly defining function for $\mathcal{Z}$ if

(i) $\left.B\right|_{\mathcal{Z}}=0$,

(ii) there are $n$ functions among the $B_{i}, \widetilde{B}:=\left(B_{1}, \ldots, B_{n}\right)$ say, such that for all $z \in \mathcal{Z}, \widetilde{B}=M_{z} \cdot \Phi_{z}$ for some $M_{z} \in H^{\infty} \otimes \mathbb{M}_{n}$ such that

$$
\begin{aligned}
\left\|M_{z}\right\| \leq \delta^{-1} & \text { on } \mathbb{D}^{n}, \\
\left\|M_{z}^{-1}\right\| \leq \delta^{-1} & \text { on }\left\{\left\|\Phi_{z}\right\|<\delta\right\},
\end{aligned}
$$

(iii) for each $\varepsilon>0$ there is $\eta>0$ such that

$$
\zeta \in \bigcap_{z \in \mathcal{Z}}\left\{\left|\Phi_{z}\right| \geq \varepsilon\right\} \Rightarrow|B(\zeta)| \geq \eta
$$

One says that $\widetilde{B}$ is equivalent to $\Phi_{z}$ near each $z \in \mathcal{Z}$. The meaning of uniform equivalence is clear.

Theorem 1 will be proved once we show that:

TheOREM 2. If $B$ is an $N$-strongly defining function for $\mathcal{Z}$ in $\mathbb{D}^{n}$, then $B$ is a universal divisor for $\mathcal{Z}$ in $H^{p}\left(\mathbb{D}^{n}\right)$ for $2<p<\infty$.

TheOREM 3. If $\mathcal{Z}$ is an interpolating sequence in $\mathbb{D}^{n}$, then there exists an $(n+2)$-strongly defining function for $\mathcal{Z}$.

Denote by $I(\mathcal{Z})$ the ideal of all holomorphic functions $f$ in $\mathbb{D}^{n}$ with $\left.f\right|_{\mathcal{Z}}=0$. Observe that condition (1) means that $H^{p} \cap I(\mathcal{Z})=H^{p} \cdot B$.

Corollary 1 . Assume that $\mathcal{Z}_{1}, \mathcal{Z}_{2}$ are interpolating sequences in $\mathbb{D}^{n}$. The following conditions are equivalent:

(i) $\mathcal{Z}_{1} \subset \mathcal{Z}_{2}$

(ii) $I\left(\mathcal{Z}_{2}\right) \cap H^{p} \subset I\left(\mathcal{Z}_{1}\right) \cap H^{p}$ for all $2<p<\infty$,

(iii) $I\left(\mathcal{Z}_{2}\right) \cap H^{p} \subset I\left(\mathcal{Z}_{1}\right) \cap H^{p}$ for some $2<p<\infty$,

(iv) $\left|B_{2}(z)\right| \leq C_{K}\left|B_{1}(z)\right|$, where $B_{1}, B_{2}$ are the divisors associated with $\mathcal{Z}_{1}, \mathcal{Z}_{2}$, respectively. The constant $C_{K}$ is the same for all $z$ from the compact subset $K \subset \mathbb{D}^{n}$. 
Proof. The implications (i) $\Rightarrow($ ii $) \Rightarrow($ iii) are obvious. To prove (iii) $\Rightarrow$ (iv) observe that if $B_{2}$ is the divisor for $\mathcal{Z}_{2}$, then $B_{2} \in H^{p}$ for any $1<p<\infty$ and consequently $B_{2} \mid \mathcal{Z}_{1}=0$. Therefore $B_{2}=F B_{1}$ for some $F \in H^{p} \otimes M_{n+2}$. Since evaluation at a point is continuous on $H^{p}$, we have $(i v)$.

Observe now that if we assume (iv), then $B_{2}(\zeta)=0$ for any $\zeta \in \mathcal{Z}_{1}$. Consequently, we have

$$
\zeta \in \mathbb{D}^{n} \cap \bigcap_{\varepsilon>0} \bigcup_{z \in \mathcal{Z}_{2}}\left\{\left|\Phi_{z}(\zeta)\right| \leq \varepsilon\right\}=\mathcal{Z}_{2} .
$$

Corollary 2. If $I\left(\mathcal{Z}_{1}\right) \cap H^{p}=I\left(\mathcal{Z}_{2}\right) \cap H^{p}$ for some $p$ with $2<p<\infty$, then $\mathcal{Z}_{1}=\mathcal{Z}_{2}$ whenever $\mathcal{Z}_{1}, \mathcal{Z}_{2}$ are interpolating sequences in $\mathbb{D}^{n}$.

\section{Notation}

- $\mathcal{Z}=\left\{z_{k}\right\}_{k \in \mathbb{N}}$ - a sequence in $\mathbb{D}^{3}$,

- $d A=d A_{1} d A_{2} d A_{3}$ - the area measure in $\mathbb{D}^{3}$,

- $d m=d m_{1} d m_{2} d m_{3}$ - the normalized Lebesgue measure on $\mathbb{T}^{3}$,

- $d \Omega=d \Omega_{1} d \Omega_{2} d \Omega_{3}$,

- $d \Omega_{i}=\log \frac{1}{\left|z_{i}\right|} d A_{i}, i=1,2,3$,

- $\mathcal{E}$ - the space of all differential forms with coefficients from $C^{\infty}\left(\overline{\mathbb{D}}^{3}\right)$,

- $\mathcal{E}_{q}$ - the space of all $(0, q)$-differential forms with coefficients from $C^{\infty}\left(\overline{\mathbb{D}}^{3}\right)$

- $\Lambda^{l}$ - the exterior algebra generated by $e_{1}, \ldots, e_{l}$,

- $\cap$ - denotes also the product in $\Lambda^{l}$,

- $\Lambda_{1}^{l}$ - the linear subspace of $\Lambda^{l}$ spanned by $e_{1}, \ldots, e_{l}$,

- $\Lambda_{2}^{l}$ - the linear subspace of $\Lambda^{l}$ spanned by $e_{i} \cap e_{j}, 1 \leq i, j \leq l$,

- $H^{p}=H^{p}\left(\mathbb{D}^{n}\right)$ - the Hardy space in the polydisk,

- $C(A, B)$ - a constant which depends only on $A$ and $B$ and sometimes on $n$,

- $A \lesssim B-$ means that there exists a constant $C$ such that $A \leq C B$ and the concrete value of $C$ is of no importance for the proof.

3. Methods and proofs. Observe that if $\mathcal{Z}=\emptyset$, then the problem is actually the $H^{p}$ corona problem. On the other hand, the Gleason problem, i.e. the problem of finding, for a given function $f \in H^{p}$ with $f(0)=0$, functions $F_{1}, \ldots, F_{n} \in H^{p}$ such that $f=z_{1} F_{1}+\cdots+z_{n} F_{n}$, has a trivial solution in the polidysk (and the standard solution in the case of the unit ball). The main idea behind the proof in [3] is to make use of these two observations. We adapt this construction to the setting of the polydisk.

More specifically, the first step is to solve the $H^{p}$ corona problem off some neighbourhood of $\mathcal{Z}$. What is important here is that we have to deal essentially with the problem for $H^{\infty}$ functions. Then we solve the Gleason problem at $0 \in \mathbb{D}^{n}$ and use the fact that the automorphisms of $\mathbb{D}^{n}$ act 
transitively to obtain the solution in a neighbourhood of $\mathcal{Z}$. The next step is to glue these two solutions together in a standard way. Analysis of the corresponding Koszul complex leads to some $\bar{\partial}$-problem in $\mathbb{D}^{n}$. The scheme of solving the equations which one encounters here was described in [7]. This involves solving higher order $\bar{\partial}$-equations in the spirit of Wolff's proof of the corona theorem and the technique which is sometimes called the projection method for the polydisk.

As for the "language" of the proof, we keep the notation introduced in [4]. This makes the proofs not only much more transparent, but also easy to generalize to higher dimensions. The style of the paper is rather concise, due to the fact that the core of the construction is the same as in [3]. The reader is invited to consult the papers [1], [3], [4], [5], [7] for the details omitted here.

$\mathcal{E} \otimes \Lambda^{l}$ is an algebra under the multiplication

$$
\left(\sum_{I} \gamma_{I} e^{I}\right) \cap\left(\sum_{J} \delta_{J} e^{J}\right):=\sum_{I, J} \gamma_{I} \wedge \delta_{J} e^{I} \cap e^{J},
$$

where $\cap$ on the right hand side stands for the product in the exterior algebra $\Lambda^{l}$, while $\wedge$ is the wedge product in $\mathcal{E}$. Assume now that $B$ is an $N$ strongly defining function for $\mathcal{Z}$. We write $B=B_{1} e_{1}+\cdots+B_{N} e_{N}$ and introduce the operator $\delta_{B}$ on $\mathcal{E} \otimes \Lambda^{N}$ by $\delta_{B} f=B \cup f$. The bilinear operator $\cup$, the inner multiplication in $\Lambda^{l}$, is defined on the generators in the following way:

$$
\begin{aligned}
& e_{i} \cup\left(e_{i_{1}} \cap \cdots \cap e_{i_{m}}\right) \\
& \quad= \begin{cases}(-1)^{i_{j}+1} e_{i_{1}} \cap \cdots \cap \widehat{e}_{i_{j}} \cap \cdots \cap e_{m} & \text { if } i_{j}=i, \\
0 & \text { if } i_{j} \neq i \text { for } 1 \leq j \leq m,\end{cases}
\end{aligned}
$$

and is extended in an obvious way to $\mathcal{E} \otimes \Lambda^{l}$. Similarly, we extend $\bar{\partial}$ to $\mathcal{E} \otimes \Lambda$ (cf. [4]).

The Koszul complex, i.e. $\mathcal{E} \otimes \Lambda$ equipped with $\bar{\partial}$ and $\delta_{B}$, is a double differential complex. This means that $\bar{\partial}^{2}=\delta_{B}^{2}=0$ and $\bar{\partial} \delta_{B}=\delta_{B} \bar{\partial}$, since $B=B_{1} e_{1}+\cdots+B_{N} e_{N}$ is holomorphic.

Apart from $\bar{\partial}$ we also use the operators $\bar{\partial}_{1}, \bar{\partial}_{2}, \bar{\partial}_{3}: \mathcal{E}_{q} \otimes \Lambda \rightarrow \mathcal{E}_{q+1} \otimes \Lambda$.

First we prove the existence of a strongly defining function for $\mathcal{Z}$. Actually, it is enough to mimic the argument from [3]. This is the reason why we only sketch it. However, at some point we will be slightly more careful.

Proof of Theorem 3. Assume that $\mathcal{Z}$ is an interpolating sequence in the polydisk. To prove the existence of a strongly defining function for $\mathcal{Z}$ one has to check whether the construction from [3] works in the setting of the polydisk.

The first step is to show that for any finite interpolating sequence $S \subset \mathbb{D}^{n}$ with $|S|=m$, there exist functions $\beta_{j}^{m} \in H^{\infty}\left(\mathbb{D}^{n}\right), j=1, \ldots, m$, such that 
$\beta_{j}^{m}\left(z_{k}\right)=\delta_{j k}$ and

$$
\sum_{j=1}^{m}\left|\beta_{j}^{m}(z)\right|^{2} \leq C^{2}
$$

with a constant $C$ independent of $n, z$ and $m$. The argument is the same as in [1] and is independent of the domain.

This implies that if $\mathcal{Z}$ is an interpolating sequence in $\mathbb{D}^{n}$, then there exists a sequence $\left(\beta_{j}\right)_{j \in \mathbb{N}}$ of $H^{\infty}$ functions with $\beta_{j}\left(z_{k}\right)=\delta_{j k}$ such that

$$
\sum_{j=1}^{\infty}\left|\beta_{j}(z)\right|^{2} \leq C^{2}
$$

with the same constant. To show this one considers the Banach space $H^{\infty}\left(\mathbb{D}^{n}, l^{2}\right)$ consisting of bounded holomorphic functions on $\mathbb{D}^{n}$ with values in $l_{2}$ and the norm

$$
\|f\|_{H^{\infty}\left(\mathbb{D}^{n}, l^{2}\right)}:=\sup _{z \in \mathbb{D}^{n}}\left(\sum_{k=1}^{\infty}\left|f_{k}(z)\right|^{2}\right)^{1 / 2} .
$$

Since this is a dual space, the sequence $\left(\beta_{1}^{1}, 0,0, \ldots\right),\left(\beta_{1}^{2}, \beta_{2}^{2}, 0, \ldots\right), \ldots$ has a weak-* convergent subsequence. Denote by $\beta=\left(\beta_{j}\right)_{j \in \mathbb{N}}$ the weak- $*$ limit of this subsequence. Naturally, $\beta$ satisfies (2) and $\beta_{j}\left(z_{k}\right)=\delta_{j k}$ for $j, k \in \mathbb{N}$. Indeed, weak-* convergence implies that for each $j$, the sequence $\beta_{j}^{n}$ converges pointwise.

To conclude the proof of the theorem, it is enough to define

$$
\begin{gathered}
H_{k}=\beta_{k} \prod_{i \neq k}\left(1-\beta_{i}\right), \quad B_{i}=\sum_{k} H_{k} \Phi_{z_{k}}^{i}, \quad 1 \leq i \leq n, \\
B_{n+1}=\prod_{k}\left(1-\beta_{k}\right), \quad B_{n+2}=\prod_{k}\left(1-H_{k}\right) .
\end{gathered}
$$

Observe that $\sum_{k=1}^{\infty}\left|H_{k}(z)\right| \leq C$, with the constant independent of $z$. The proof of Theorem 2.2 in [3] will show that we have constructed a strongly defining function for $\mathcal{Z}$ of dimension $n+2$, once we prove the next lemma.

LEMMA 1. Let $H \in H^{\infty}\left(\mathbb{D}^{n}, l^{1}\right)$ and assume that $H(z)=0$ for some $z \in \mathbb{D}^{n}$. Then there exists $\alpha \in H^{\infty}\left(\mathbb{D}^{n}, l^{1}\right) \otimes \Lambda_{1}^{N}$ such that $H=\alpha \cup \Phi_{z}$ and $\|\alpha\|_{H^{\infty}\left(\mathbb{D}^{n}, l^{1}\right) \otimes \Lambda_{1}^{n}} \leq C\|H\|_{H^{\infty}\left(\mathbb{D}^{n}, l^{1}\right)} \cdot$

Proof. Write $H=\left(H_{1}, H_{2}, \ldots\right)$. When $z=0$ we may write, for each $k$,

$$
\begin{aligned}
H_{k}(z) & =z_{n} \frac{H_{k}\left(z_{1}, \ldots, z_{n}\right)-H_{k}\left(z_{1}, \ldots, z_{n-1}, 0\right)}{z_{n}}+\cdots+z_{1} \frac{H_{k}\left(z_{1}, 0, \ldots, 0\right)}{z_{1}} \\
& =z_{1} \alpha_{k 1}+\cdots+z_{n} \alpha_{k n},
\end{aligned}
$$

with trivial estimates showing that indeed $\|\alpha\|_{H^{\infty}\left(\mathbb{D}^{n}, l^{1}\right) \otimes \Lambda_{1}^{N}} \leq C\|H\|_{H^{\infty}\left(\mathbb{D}^{n}, l^{1}\right)}$. The general case follows by composition with automorphisms of $\mathbb{D}^{n}$. 
The same argument would actually show that for an $H^{p}$ function $f$, $1<p<\infty$, with $f(z)=0$ for some $z \in \mathbb{D}^{n}$ there exists $F_{z} \in H^{p} \otimes \Lambda_{1}^{n}$ such that $f=F_{z} \cup \Phi_{z}$ and $\left\|F_{z}\right\|_{H^{p} \otimes \Lambda_{1}^{n}} \lesssim\|f\|_{H^{p}}$. However, we need $F_{z}$ with these properties to be defined in a different manner (cf. Proposition 4.2 in [3] and Lemma 6 below).

Fix now a number $\delta>0$. Its value follows from the proofs below. Let $\chi: \mathbb{R}_{+} \rightarrow[0,1]$ be a smooth cut-off function satisfying $\chi(t)=1$ for $t<1 / 2$ and $\chi(t)=0$ if $t>1$. Define $\gamma:=G+\left(1-\chi_{\mathcal{Z}}\right) H$, where $H=f b$ and

$$
b=\sum_{j=1}^{N} \frac{\bar{B}_{j}}{|B|^{2}} e_{j}
$$

is defined only on the set $\{|B|>0\}$, and

$$
G=\sum_{z \in \mathcal{Z}} \chi\left(\frac{\left|\Phi_{z}\right|^{2}}{\delta^{2}}\right) G_{z}, \quad G_{z}=\sum_{j=1}^{n}\left({ }^{t} M_{z}^{-1} F_{z}\right)_{j} e_{j} .
$$

Define $\chi_{\mathcal{Z}}$ to be the function

$$
\sum_{z \in \mathcal{Z}} \chi_{z}(\cdot):=\sum_{z \in \mathcal{Z}} \chi\left(\frac{\left|\Phi_{z}(\cdot)\right|^{2}}{\delta^{2}}\right) .
$$

Observe that for each $\varepsilon>0, b$ is bounded in $\{|B|>\varepsilon\}$. Let $\omega^{0}=\gamma$ and

$$
\omega^{r}=b \cap \bar{\partial} \omega^{r-1}=b \cap(\bar{\partial} b)^{r-1} \cap \bar{\partial} \gamma
$$

for $r>0$. Observe that $\omega^{r}$ is well-defined in $\mathbb{D}^{n}$. Indeed, in the set $\bigcup_{z \in \mathcal{Z}}\left\{\left|\Phi_{z}\right|^{2}<\delta^{2} / 2\right\}$ we have $\bar{\partial} \gamma=0$. By condition (iii) of the definition of the defining function, this implies that the support of $\omega^{r}$ is contained in the set $\{|B|>\varepsilon\}$ for some non-zero $\varepsilon$. Furthermore, taking $\gamma_{s}(z):=\gamma(s z)$ and $b_{s}$ for $s<1$, we may assume that $\omega^{r}$ belongs to $\mathcal{E}_{r-1} \otimes \Lambda_{r+1}$.

Lemma 2. Let $K$ be any (not necessarily linear) $\bar{\partial}$-solving operator. Define

$$
\begin{aligned}
\eta & =\omega^{0}-\delta_{B} K\left(\omega^{1}-\delta_{B} K\left(\omega^{2}-\cdots\right)\right) \\
& =\omega^{0}-\delta_{B} K\left(\omega^{1}-\delta_{B} K\left(\omega^{2}-\cdots-\delta_{B} K\left(\omega^{n-1}-\delta_{B} K \omega^{n}\right) \ldots\right)\right) .
\end{aligned}
$$

Then $\bar{\partial} \eta=0$ and $\delta_{B} \eta=f$.

Proof. First of all observe that $\delta_{B} \eta=\delta_{B} \omega^{0}=f$. As for the first property notice that

$$
\begin{aligned}
\bar{\partial} \eta & =\bar{\partial} \omega^{0}-\delta_{B} \bar{\partial} K\left(\omega^{1}-\delta_{B} K\left(\omega^{2}-\cdots\right)\right) \\
& =\bar{\partial} \omega^{0}-\delta_{B} \omega_{1}+\delta_{B}^{2} K\left(\omega^{2}-\cdots\right) \\
& =\bar{\partial} \omega^{0}-\delta_{B}\left(b \cap \bar{\partial} \omega^{0}\right)=0,
\end{aligned}
$$


since $\delta_{B} \bar{\partial} \gamma=\bar{\partial} \delta_{B} \gamma=\bar{\partial} f=0$. For this argument to work we must show that $\omega^{1}-\delta_{B} K\left(\omega^{2}-\cdots\right)$ is $\bar{\partial}$-closed. Observe that

$\bar{\partial}\left(\omega^{1}-\delta_{B} K\left(\omega^{2}-\cdots\right)\right)=\bar{\partial} \omega^{1}-\delta_{B} \bar{\partial} K\left(\omega_{2}-\delta_{B} K(\ldots)\right)=\bar{\partial} \omega^{1}-\delta_{B} \omega^{2}=0$

if $\omega_{2}-\delta_{B} K(\ldots)$ is $\bar{\partial}$-closed. Thus, an induction argument completes the proof.

In other words, $\eta$ is holomorphic and satisfies $B_{1} \eta_{1}+\cdots+B_{N} \eta_{N}=f$. What still has to be proved is that $\eta$ may be chosen to belong to $H^{p} \otimes \Lambda_{1}^{l}$. To show this, one first solves the problem for $B_{s}$, with $s<1$, finding appropriate $\eta_{s}$ satisfying $\left\|\eta_{s}\right\|_{H^{p}} \leq C$ with bound independent of $s$. A normal family argument gives the existence of $\eta \in H^{p} \otimes \Lambda_{1}^{l}$ such that $\delta_{B} \eta=f$.

We will restrict our attention to the case $n=3$. The general case can be proved analogously. Lemma 2 shows that the problem in $\mathbb{D}^{3}$ amounts to solving the following equations in $\mathbb{C}^{3}$ :

$$
\begin{aligned}
& \bar{\partial} \eta^{3}=\omega^{3}, \\
& \bar{\partial} \eta^{2}=\omega^{2}-\delta_{B} \eta^{3}, \\
& \bar{\partial} \eta^{1}=\omega^{1}-\delta_{B} \eta^{2} .
\end{aligned}
$$

Let us recall the concept of a Carleson measure in the polydisk [5]. For $z=r e^{i \theta_{0}}$, let $I_{z}$ denote the arc $\left\{e^{i \theta}:\left|\theta-\theta_{0}\right|<1-r\right\}$. Let $U$ be any open connected subset of $\mathbb{T}^{n}$. Set $S(U):=\left\{z \in \mathbb{D}^{n}: I_{z_{1}} \times \cdots \times I_{z_{n}} \subset U\right\}$. Equivalently, $S(U)=\bigcup_{I_{1} \times \cdots \times I_{n} \subset U} S\left(I_{1}\right) \times \cdots \times S\left(I_{n}\right)$. We shall consider positive measures $\mu$ on $\mathbb{D}^{n}$ satisfying $\mu(S(U)) \lesssim|U|$. It was indicated in [5] that this is the correct generalization of the notion of Carleson measure for the polydisk. This means for instance that such a measure is bounded on the $L^{p}\left(\mathbb{T}^{n}\right)$ space [5], i.e.

$$
\left(\int_{\mathbb{D}^{n}}|F|^{p} d \mu\right)^{1 / p} \leq\left(\int_{\mathbb{T}^{n}}|f|^{p} d m\right)^{1 / p}
$$

for any $f \in L^{p}, 1<p<\infty$. Here $F$ stands for the $n$-harmonic extension of $f$. Therefore, such measures are called Carleson measures on $\mathbb{D}^{n}$.

Additionally, we consider positive measures on $\mathbb{D}^{n}$ satisfying $\mu(S(U)) \lesssim$ $|U|^{\alpha}$ for some $0<\alpha \leq 1$ and call them Carleson measures of order $\alpha$, or simply $\alpha$-Carleson measures (cf. [2]).

If $f$ is an $L^{p}\left(\mathbb{T}^{n}\right)$ function, then $\tilde{f}$ stands for the Poisson extension of $|f|$. We abuse the notation in an accepted manner and write $\widetilde{f}$ also if $f \in H^{p}$.

Proposition 1.

(i) There exists $\eta^{3} \in \mathcal{E}_{2} \otimes \Lambda^{N}$ satisfying

$$
\bar{\partial} \eta^{3}=\omega^{3}
$$


such that

$$
\eta^{3}=\bar{\partial}_{1} \bar{\partial}_{2} u_{12}+\bar{\partial}_{1} \bar{\partial}_{3} u_{13}+\bar{\partial}_{2} \bar{\partial}_{3} u_{23}
$$

and $\left\|u_{12}\right\|_{L^{p}},\left\|u_{13}\right\|_{L^{p}},\left\|u_{23}\right\|_{L^{p}} \leq C$, with a constant which depends on $\|B\|_{H^{\infty} \otimes \Lambda_{1}^{N}},\|f\|_{L^{p}}$ and $\|\widetilde{f}\|_{L^{p}}$ but not on $s$.

(ii) There exists $\eta^{2} \in \mathcal{E}_{1} \otimes \Lambda^{N}$ satisfying

$$
\bar{\partial} \eta^{2}=\omega^{2}-\delta_{B} \eta^{3}
$$

such that

$$
\eta^{2}=\bar{\partial}_{1} u_{1}+\bar{\partial}_{2} u_{2}+\bar{\partial}_{3} u_{3}
$$

with $\left\|u_{1}\right\|_{L^{p}},\left\|u_{2}\right\|_{L^{p}},\left\|u_{3}\right\|_{L^{p}} \leq C\left(\delta,\|B\|_{H^{\infty} \otimes \Lambda_{1}^{N}},\|f\|_{L^{p}},\|\widetilde{f}\|_{L^{p}}\right)$.

(iii) There exists $\eta^{1} \in \mathcal{E}_{0} \otimes \Lambda^{N}$ satisfying

$$
\begin{gathered}
\bar{\partial} \eta^{1}=\omega^{1}-\delta_{B} \eta^{2} \\
\text { with }\left\|\eta^{1}\right\|_{L^{p}} \lesssim C\left(\delta,\|B\|_{H^{\infty} \otimes \Lambda_{1}^{N}},\|f\|_{L^{p}},\|\widetilde{f}\|_{L^{p}}\right)
\end{gathered}
$$

Observe that Proposition 1(iii) together with a normal family argument gives the proof of Theorem 2 .

Proof of Proposition 1(i). It is enough to solve the equation $\bar{\partial}_{1} \bar{\partial}_{2} \bar{\partial}_{3} u=$ $\omega^{3}$ and define $u_{12}=u, u_{13}=0, u_{23}=0$. Thus, Proposition $1(\mathrm{i})$ is a consequence of the following lemma:

Lemma 3. Suppose $g \in C^{3}\left(\overline{\mathbb{D}}^{3}\right)$ satisfies:

(i) $|g|^{2} d \Omega$ is an $\alpha=1-2 / p$-Carleson measure,

$$
\begin{gathered}
\int_{\mathbb{D}^{3}}|h| \cdot\left|\partial_{1} \partial_{2} \partial_{3} g\right| d \Omega \lesssim 1, \\
\sum_{i \neq j, j \neq k, i \neq k} \int_{\mathbb{D}^{3}}\left|\partial_{i} h\right| \cdot\left|\partial_{j} \partial_{k} g\right| d \Omega \lesssim 1, \\
\sum_{i \neq j, j \neq k, i \neq k} \int_{\mathbb{D}^{3}}\left|\partial_{i} \partial_{j} h\right| \cdot\left|\partial_{k} g\right| d \Omega \lesssim 1,
\end{gathered}
$$

for any $h \in H^{q}$ with $\|h\|_{L^{q}}=1$. Then the equation

$$
\frac{\partial^{3} u}{\partial \bar{z}_{1} \partial \bar{z}_{2} \partial \bar{z}_{3}}=g
$$

has a solution $u \in C^{\infty}\left(\overline{\mathbb{D}}^{3}\right)$ with $\|u\|_{L^{p}\left(\mathbb{T}^{3}\right)} \leq C$.

Proof. Let $u$ be a $C^{\infty}\left(\overline{\mathbb{D}}^{3}\right)$ solution to $(7)$. Any $C^{\infty}\left(\overline{\mathbb{D}}^{3}\right)$ solution is of the form $u+v$, where $v$ belongs to $C^{\infty}\left(\overline{\mathbb{D}}^{3}\right)$ and is $\bar{\partial}_{1} \bar{\partial}_{2} \bar{\partial}_{3}$-closed. By duality, we have

$$
\inf _{v \in H^{p}\left(\mathbb{D}^{3}\right)}\|u+v\|_{L^{p}} \leq \sup \left\{\left|\int_{\mathbb{T}^{3}} u h d m\right|: h \in H^{q},\|h\|_{H^{q}} \leq 1\right\} .
$$


For a fixed $h$, by Green's theorem, we have

$$
\left|\int_{\mathbb{T}^{3}} u h d m\right|=c\left|\int_{\mathbb{D}^{3}} \Delta_{1} \Delta_{2} \Delta_{3}(u h) d \Omega\right|=c\left|\int_{\mathbb{D}^{3}} \partial_{1} \partial_{2} \partial_{3}(h g) d \Omega\right| .
$$

It is enough to estimate the right-hand side of the above equality by $\|h\|_{H^{q}}$. It follows from assumptions (ii)-(iv) that we have to deal with the expression involving $|g| d \Omega$ only. We will work in $\mathbb{R}_{+}^{2} \times \mathbb{R}_{+}^{2} \times \mathbb{R}_{+}^{2}$. For $x \in \mathbb{R}^{3}$ let $\Gamma(x)=$ $\Gamma\left(x_{1}\right) \times \Gamma\left(x_{2}\right) \times \Gamma\left(x_{3}\right)$ denote the product cone. For a function $h \in H^{1}$ define the function

$$
A h(x)=\left(\int_{\Gamma(x)}\left|\frac{\partial^{3} h}{\partial z_{1} \partial z_{2} \partial z_{3}}\right|^{2} d A\right)^{1 / 2}
$$

and sets $O_{j}=\left\{x \in \mathbb{R}^{3}: A h(x)>2^{j}\right\}$. Let $F_{j}$ denote the set of all points $z \in \mathbb{R}_{+}^{2} \times \mathbb{R}_{+}^{2} \times \mathbb{R}_{+}^{2}$ such that $\left|R_{z} \cap O_{j}\right|>\left|R_{z}\right| / 2$ and $\left|R_{z} \cap O_{j+1}\right| \leq\left|R_{z}\right| / 2$, where $R_{z}$ is the rectangle centred at $\left(x_{1}, x_{2}, x_{3}\right)$ with side lengths $2 y_{1}, 2 y_{2}$ and $2 y_{3}$, respectively. Define also $\Omega_{j}=\bigcup_{z \in F_{j}} R_{z}$.

Obviously (cf. [7] and [8]), we have

$$
\begin{array}{r}
\int_{\mathbb{R}_{+}^{2} \times \mathbb{R}_{+}^{2} \times \mathbb{R}_{+}^{2}}\left|\frac{\partial^{3} h}{\partial z_{1} \partial z_{2} \partial z_{3}}\right||g| y_{1} y_{2} y_{3} d x d y=\sum_{j} \int_{F_{j}}\left|\frac{\partial^{3} h}{\partial z_{1} \partial z_{2} \partial z_{3}}\right||g| y_{1} y_{2} y_{3} d x d y \\
\leq \sum_{j}\left(\int_{F_{j}}\left|\frac{\partial^{3} h}{\partial z_{1} \partial z_{2} \partial z_{3}}\right|^{2} y_{1} y_{2} y_{3} d x d y\right)^{1 / 2}\left(\int_{F_{j}}|g|^{2} y_{1} y_{2} y_{3} d x d y\right)^{1 / 2}
\end{array}
$$

which can be estimated by (recall that $2<p<\infty$ )

$$
\begin{aligned}
& \left(\int_{\mathbb{R}_{+}^{2} \times \mathbb{R}_{+}^{2} \times \mathbb{R}_{+}^{2}}|g|^{2} y_{1} y_{2} y_{3} d x d y\right)^{1 / p} \\
& \quad \times\left(\sum_{j}\left|O_{j}\right|^{q(1 / 2-1 / p)}\left(\int_{\Omega_{j} \backslash O_{j+1}} \int_{\Gamma(x)}\left|\frac{\partial^{3} h}{\partial z_{1} \partial z_{2} \partial z_{3}}\right|^{2} d x d y\right)^{q / 2}\right)^{1 / q} \\
& \leq\left(\sum_{j} 2^{q(j+1)}\left|O_{j}\right|^{q / 2} \cdot\left|O_{j}\right|^{q(1 / 2-1 / p)}\right)^{1 / q} \lesssim\|h\|_{H^{q}} .
\end{aligned}
$$

The last inequality is a consequence of results in [10] (again $2<p<\infty$ ).

Let us recall that, by the results in [5], if both $|g|^{2} d \Omega$ and $\left|\partial_{1} \partial_{2} \partial_{3} g\right| d \Omega$ are Carleson measures, and the inequalities

$$
\begin{aligned}
& \sum_{i \neq j, j \neq k, i \neq k} \int_{\mathbb{D}^{3}}\left|\partial_{i} f\right| \cdot\left|\partial_{j} \partial_{k} g\right| d \Omega \lesssim 1, \\
& \sum_{i \neq j, j \neq k, i \neq k} \int_{\mathbb{D}^{3}}\left|\partial_{i} \partial_{j} f\right| \cdot\left|\partial_{k} g\right| d \Omega \lesssim 1
\end{aligned}
$$


hold for any $H^{1}$ function $f$ of norm 1 , then the equation (7) is solvable with sup-norm control of the solution.

The symbol $\mathfrak{D}$ will denote any constant coefficient differential operator (c.c.d.o.) (differentiation with respect to $z_{1}, z_{2}, z_{3}$ ). We write $\mathfrak{D}^{i}$ for a c.c.d.o. of order $\leq i$.

Lemma 4. Assume that $F_{1}, F_{2}, F_{3}$ are bounded, holomorphic functions in $\mathbb{D}^{3}$. Measures of the form

$$
\left|\mathfrak{D}^{i_{1}} F_{1} \mathfrak{D}^{i_{2}} F_{2} \mathfrak{D}^{i_{3}} F_{3}\right|^{2} d \Omega
$$

satisfy the Carleson condition on $\mathbb{D}^{3}$, provided $0 \leq i_{1}+i_{2}+i_{3} \leq 3$.

Proof. We will deal only with

$$
\left|\partial_{1} F_{1}\right|^{2}\left|\partial_{2} F_{2}\right|^{2}\left|\partial_{3} F_{3}\right|^{2} d \Omega
$$

where $F_{1}, F_{2}, F_{3}$ are bounded and holomorphic in $\mathbb{D}^{3}$.

Let $U$ be an open connected subset of $\mathbb{T}^{2}$. We will first show that the measure

$$
\mu=\left|\partial_{1} F_{1}\right|^{2}\left|\partial_{2} F_{2}\right|^{2} \log \frac{1}{\left|z_{1}\right|} \log \frac{1}{\left|z_{2}\right|} d A
$$

is a Carleson measure on $\mathbb{D}^{2}$, i.e. $\mu(S(U)) \lesssim|U|$. Let us recall a decomposition from [5]. Let $I$ be the projection of $U$ onto the first variable, and $J$ onto the second variable. Fix $x_{1} \in I$ and $0 \leq y_{1} \leq d\left(x_{1}, I^{\mathrm{c}}\right)$. When $y_{1}>0$, let $\left\{J_{x_{1}, y_{1}, l}\right\}_{l}$ denote the collection of maximal disjoint intervals contained in $J$ such that $\left(x_{1}-y_{1}, x_{1}+y_{1}\right) \times J_{x_{1}, y_{1}, l} \subset U$. To prove that $\mu$ is a Carleson measure, it is enough to show that $\left|\partial_{1}\left(F_{1} \partial_{2} F_{2}\right)\right|^{2} d \Omega$ is a Carleson measure, since by the results in [5] the measure $\left|\partial_{1} \partial_{2} F_{2}\right|^{2} d \Omega$ has this property. Following [5] (cf. also [6]) one obtains

$$
\begin{aligned}
& \int_{S(U)}\left|\partial_{2}\left(F_{2} \partial_{1} F_{1}\right)\right|^{2} y_{2} d y_{2} d x_{2} y_{1} d y_{1} d x_{1} \\
& \leq\left(2+\frac{C}{\alpha_{0}^{1 / 2}}+\sum_{m=1}^{\infty} \alpha_{m-1}\left(2+\frac{C}{\alpha_{m}^{1 / 2}}\right)\right)|U|\left\|F_{1}\right\|_{\infty}^{2}\left\|F_{2}\right\|_{\infty}^{2},
\end{aligned}
$$

which yields the conclusion, if the sequence $\alpha_{m}$ has been chosen properly.

If $U$ is an open connected subset of $\mathbb{T}^{3}$, then one considers a similar decomposition. Namely, let $V$ be the projection of $U$ onto the first two variables. Then $V$ is open and connected. Let $I$ be the projection of $V$ onto the first variable. For each $0 \leq y_{1} \leq d\left(x_{1}, I^{\mathrm{c}}\right)$ choose a maximal family $\left\{J_{x_{1}, y_{1}, l}\right\}_{l}$ of open intervals such that $\left(x_{1}-y_{1}, x_{1}+y_{1}\right) \times J_{x_{1}, y_{1}, l} \subset U$. For any $x_{2} \in \bigcup_{l} J_{x_{1}, y_{1}, l}$ and $0 \leq y_{2} \leq d\left(x_{2},\left(J_{x_{1}, y_{1}, l}^{x_{2}}\right)^{\mathrm{c}}\right)$ choose a maximal family of intervals such that $\left(x_{1}-y_{1}, x_{1}+y_{1}\right) \times\left(x_{2}-y_{2}, x_{2}+y_{2}\right) \times K_{x_{1}, y_{1}, x_{2}, y_{2}, l, m} \subset U$. 
The proof can be completed as in [5]. We omit the rather technical details.

Observe that

$$
\omega^{3}=f b \cap(\bar{\partial} b)^{2} \cap \bar{\partial}\left(\left(1-\chi_{Z}\right) b\right)+b \cap(\bar{\partial} b)^{2} \cap \bar{\partial} G .
$$

Thus, there exists a $\xi$ with $\|\xi\|_{L^{\infty}} \lesssim 1$ such that $\bar{\partial}_{1} \bar{\partial}_{2} \bar{\partial}_{3} \xi=b \cap(\bar{\partial} b)^{2} \cap$ $\bar{\partial}\left(\left(1-\chi_{\mathcal{Z}}\right) b\right)$ - this follows from the results in [7], which we have already cited. Consequently, $\bar{\partial}_{1} \bar{\partial}_{2} \bar{\partial}_{3}(f \xi)=f b \cap(\bar{\partial} b)^{2} \cap \bar{\partial}\left(\left(1-\chi_{\mathcal{Z}}\right) b\right)$ and to complete the proof of Proposition 1(i) it is enough to show that $b \cap(\bar{\partial} b)^{2} \cap \bar{\partial} G$ can be written as a sum of expressions satisfying the assumptions of Lemma 3.

Before we show this fact, we formulate a couple of observations.

Lemma 5. There exists a function $D \in H^{\infty}\left(\mathbb{D}^{3}\right)$, which is equivalent to $\Phi_{z}$ near each $z \in \mathcal{Z}$, such that

$$
\left|\mathfrak{D} \Phi_{z}\right| \lesssim\left|\mathfrak{D}^{\prime} D\right|
$$

on the set $\left\{\left|\Phi_{z}\right|<\delta\right\}, z \in \mathcal{Z}$. Consequently,

$$
\sum_{z \in \mathcal{Z}}\left|\mathfrak{D} \chi_{z}\right| \lesssim\left|\mathfrak{D}^{\prime} D\right|
$$

Here $\mathfrak{D}^{\prime}$ stands for a c.c.d.o. of order not greater than the order of $\mathfrak{D}$.

Proof. Define a function $D$ by

$$
D:=\sum_{k=1}^{\infty} \Phi_{z_{k}} \beta_{k}^{2} .
$$

The fact that $D$ is equivalent to $\Phi_{z}$ near $z \in \mathcal{Z}$ can be shown as in [1]. Direct computations show (9). Indeed, first one shows that $\left|\mathfrak{D} m_{z}\right| \lesssim\left|\mathfrak{D} \Phi_{z}\right|$ in $\left\{\left|\Phi_{z}\right|<\delta\right\}$, and then uses the formula $D=m_{z} \Phi_{z}$, which holds in $\left\{\left|\Phi_{z}\right|<\delta\right\}$, to prove (9) inductively. Here $m_{z}$ stands for an $H^{\infty}$ matrix which is uniformly invertible near $z$.

The other inequality follows from the fact that the sets $\left\{\left|\Phi_{z_{1}}\right|<\delta\right\}$ and $\left\{\left|\Phi_{z_{2}}\right|<\delta\right\}$ are disjoint provided $z_{1} \neq z_{2}$.

Lemma 6. Denote by $\tilde{f}$ the Poisson extension of $|f|$. Then

$$
\left|\mathfrak{D} F_{z}(\zeta)\right| \lesssim \widetilde{f}(z) \lesssim \widetilde{f}(\zeta)
$$

for $\zeta \in\left\{\left|\Phi_{z}\right|<\delta\right\}$.

Proof. The proof is left to the reader (cf. [3]).

The fact that $\left|b \cap(\bar{\partial} b)^{2} \cap \bar{\partial} G\right|^{2} d \Omega$ is an $(1-2 / p)$-Carleson measure is now almost obvious. Indeed, each term which appears in $\left|b \cap(\bar{\partial} b)^{2} \cap \bar{\partial} G\right|^{2} d \Omega$ 
is of the form (or can be estimated by)

$$
\left|F \partial_{1} F_{1} \partial_{2} F_{2} \partial_{3} F_{3}\right|^{2} d \Omega
$$

where $F_{1}, F_{2}, F_{3}$ are bounded holomorphic functions in $\mathbb{D}^{3}$ and $F$ is a Poisson extension of an $L^{p}$ function with $2<p<\infty$.

Take now any $H^{q}$ function $h$ and observe that

$$
\begin{aligned}
\int_{\mathbb{D}^{3}}\left|\partial_{2} \partial_{3} h\right||\widetilde{f}|\left|\partial_{1} D\right|^{2}\left|\partial_{2} D\right|\left|\partial_{3} D\right| d \Omega & \\
& \lesssim \iint_{\mathbb{D}^{2} \mathbb{T}}\left|\partial_{2} \partial_{3} h\right||\widetilde{f}|\left|\partial_{2} D\right|\left|\partial_{3} D\right| d m_{1} d \Omega_{2} d \Omega_{3}=: I,
\end{aligned}
$$

because $\left|\partial_{1} D\right|^{2} \log \frac{1}{\left|z_{1}\right|} d A_{1}$ is Carleson measure on the unit disk with a Carleson norm bounded by $\|D\|_{L^{\infty}}^{2}$ and $\widetilde{f} \partial_{2} \partial_{3} h\left(\cdot, z_{2}, z_{3}\right)$ belongs to $h^{1}$ for each fixed $z_{2}$ and $z_{3}$. Thus

$$
\begin{aligned}
I & \lesssim \int_{\mathbb{T}}\left(\int_{\mathbb{T}^{2}}|h|^{q} d m_{2} d m_{3}\right)^{1 / q}\left(\int_{\mathbb{D}^{2}}|\widetilde{f}|^{2}\left|\partial_{2} D\right|^{2}\left|\partial_{3} D\right|^{2} d \Omega_{2} d \Omega_{3}\right)^{1 / p} d m_{1} \\
& \lesssim \int_{\mathbb{T}}\left(\int_{\mathbb{T}^{2}}|h|^{q} d m_{2} d m_{3}\right)^{1 / q}\left(\int_{\mathbb{T}^{2}}|\widetilde{f}|^{2} d m_{2} d m_{3}\right)^{1 / p} d m_{1} \leq\|h\|_{H^{q}}\|\widetilde{f}\|_{L^{2}}^{2 / p} .
\end{aligned}
$$

This follows from the fact that $\left|\partial_{2} D\right|^{2}\left|\partial_{3} D\right|^{2} d \Omega_{2} d \Omega_{3}$ is a Carleson measure on the bidisk with Carleson norm uniformly bounded for $z_{1} \in \mathbb{D}$ (see the proof of Lemma 3). Similar arguments also show that

$$
\begin{aligned}
& \int_{\mathbb{D}^{3}}\left|\partial_{3} h\right||\widetilde{f}|\left|\partial_{1} D\right|^{2}\left|\partial_{2} D\right|^{2}\left|\partial_{3} D\right| d \Omega \lesssim\|h\|_{H^{q}} \\
& \int_{\mathbb{D}^{3}}\left|\partial_{1} \partial_{2} \partial_{3} h\right||\widetilde{f}|\left|\partial_{1} D\right|\left|\partial_{2} D\right|\left|\partial_{3} D\right| d \Omega \lesssim\|h\|_{H^{q}}
\end{aligned}
$$

This completes the proof of Proposition 1(i).

Proof of Proposition 1(ii). Observe that

$$
\begin{aligned}
\delta_{B} \eta^{3} & =\delta_{B}\left(\bar{\partial}_{1} \bar{\partial}_{2} u_{12}+\bar{\partial}_{1} \bar{\partial}_{3} u_{13}+\bar{\partial}_{2} \bar{\partial}_{3} u_{23}\right) \\
& =\bar{\partial}_{1} \bar{\partial}_{2} \delta_{B} u_{12}+\bar{\partial}_{1} \bar{\partial}_{3} \delta_{B} u_{13}+\bar{\partial}_{2} \bar{\partial}_{3} \delta_{B} u_{23} .
\end{aligned}
$$

As a result, to complete the proof, it is enough to solve the equation

$$
\bar{\partial}_{1} \bar{\partial}_{2} v_{12}+\bar{\partial}_{1} \bar{\partial}_{3} v_{13}+\bar{\partial}_{2} \bar{\partial}_{3} v_{23}=\omega^{2}
$$

with $L^{p}$ control of the solution.

Lemma 7. Fix $1 \leq i \neq j \leq 3$ and suppose that $g \in C^{2}\left(\overline{\mathbb{D}}^{3}\right)$ satisfies:

(i) $|g|^{2} d \Omega_{i} d \Omega_{j}$ is an $\alpha=1-2 / p$-Carleson measure, 
(ii) The functions:

$$
\begin{aligned}
& \int_{\mathbb{D}^{2}}|h|\left|\partial_{i} \partial_{j} g\right| d \Omega_{i} d \Omega_{j}, \\
& \int_{\mathbb{D}^{2}}\left(\left|\partial_{i} h\right|\left|\partial_{j} g\right|+\left|\partial_{j} h\right|\left|\partial_{i} g\right|\right) d \Omega_{i} d \Omega_{j}, \\
& \int_{\mathbb{D}^{2}}\left|\partial_{i} \partial_{j} h\right||g| d \Omega_{i} d \Omega_{j}
\end{aligned}
$$

belong to $L^{1}\left(\mathbb{T}_{k}\right)$ with the norm $\lesssim\|h\|_{H^{q}}$, where $h$ is any $H^{q}$ function in $\mathbb{D}^{3}$. Then there exists $v$ such that

with $\|v\|_{L^{p}} \lesssim C$.

$$
\frac{\partial^{2} v}{\partial \bar{z}_{i} \partial z_{j}}=g
$$

Proof. Repeating the argument from Lemma 3 we are led to consider

$$
\begin{aligned}
\left|\int_{\mathbb{T}^{3}} h v d m\right| & =\left|\int_{\mathbb{T}} \int_{\mathbb{D}^{2}} \Delta_{i} \Delta_{j}(h v) d \Omega_{j} d \Omega_{i} d m_{k}\right| \\
& \leq \int\left|\int_{\mathbb{T}} \partial_{\mathbb{D}^{2}} \partial_{j}(h g) d \Omega_{i} d \Omega_{j}\right| d m_{k} .
\end{aligned}
$$

The last expression can be estimated by assumptions and using similar arguments to those in the proof of Lemma 3 by $\|h\|_{H^{q}}$. This yields the conclusion.

Observe that each term of $\omega^{2}$ satisfies the assumptions of the previous lemma. This can be shown as in the proof of Proposition 1(i). Consequently, we can solve the equation (10) with control of the $L^{p}$ norm. Lemma 3 in [7] says that we can modify $v_{12}-\delta_{B} u_{12}, v_{13}-\delta_{B} u_{13}, v_{23}-\delta_{B} u_{23}$ with control of the $L^{p}$ norm to find a solution $u_{12}, u_{13}, u_{23}$ to the equation

$$
\bar{\partial}_{1} \bar{\partial}_{2} u_{12}+\bar{\partial}_{1} \bar{\partial}_{3} u_{13}+\bar{\partial}_{2} \bar{\partial}_{3} u_{23}=\omega^{2}-\delta_{B} \eta^{3}
$$

satisfying

$$
u_{12}-u_{13}+u_{23}=0 .
$$

One checks easily that for $\eta^{2}=\bar{\partial}_{2} u_{12}+\bar{\partial}_{3} u_{13}$ we have $\bar{\partial} \eta^{2}=\omega^{2}-\delta_{B} \eta^{3}$.

Proof of Proposition 1(iii). Again we have

$$
\begin{aligned}
\delta_{B} \eta^{2} & =\delta_{B}\left(\bar{\partial}_{1} u_{1}+\bar{\partial}_{2} u_{2}+\bar{\partial}_{3} u_{3}\right) \\
& =\bar{\partial}_{1} \delta_{B} u_{1}+\bar{\partial}_{2} \delta_{B} u_{2}+\bar{\partial}_{3} \delta_{B} u_{3} .
\end{aligned}
$$

Therefore, to solve the equation $\bar{\partial} \eta^{1}=\omega^{1}-\delta_{B} \eta^{2}$ one needs to solve the equations $\bar{\partial} \eta=\omega_{1}$ only. This is achieved in two steps. First one proceeds as in the one variable case, and then one corrects the solution making use of Lemma 2 in [7]. 


\section{References}

[1] E. Amar, Interpolating sequences in the ball of $\mathbb{C}^{n}$, Ark. Mat. 38 (2000), 1-20.

[2] E. Amar et A. Bonami, Mesures de Carleson d'ordre $\alpha$ et solutions au bord de l'équation $\bar{\partial}$, Bull. Soc. Math. France 197 (1979), 23-48.

[3] E. Amar and C. Menini, Universal divisors in Hardy spaces, Studia Math. 143 (2000), 1-21.

[4] M. Andersson and H. Carlesson, Estimates of solutions of the $H^{p}$ and BMOA corona problem, Math. Ann. 316 (2000), 83-102.

[5] S.-Y. Chang, Carleson measure on a bidisc, Ann. of Math. 109 (1979), 613-620.

[6] R. Fefferman, Bounded mean oscillation on the polydisk, ibid. 110 (1979), 395-406.

[7] K.-C. Lin, $H^{p}$-solutions for the corona problem on the polydisc in $\mathbb{C}^{n}$, Bull. Sci. Math. 110 (1986), 69-84.

[8] - The $H^{p}$-corona theorem for the polydisc, Trans. Amer. Math. Soc. 341 (1994), 371-375.

[9] S. Krantz, Function Theory of Several Complex Variables, Wiley, New York, 1982.

[10] K. G. Merryfield, On the area integral, Carleson measures and $H^{p}$ in the polydisc, Indiana Univ. Math. J. 34 (1985), 663-685.

Faculty of Mathematics and Computer Science

A. Mickiewicz University

Umultowska 87

61-614 Poznań, Poland

E-mail: mjk@amu.edu.pl

Received 22.8.2006

and in final form 16.1.2007

(1694) 